NUEVAS NECESIDADES EDUCATIVAS

\title{
UNA APROXIMACIÓN A LOS CONDICIONANTES DEL SISTEMA EDUCATIVO EN ÉPOCAS DE CONFINAMIENTO SOCIAL
}

\author{
Roxana Graciela, Marsollier * \\ CONICET - Universidad Nacional de Cuyo, Argentina \\ rgmarsollier@conicet.gov.ar \\ Cristián David, Expósito ** \\ CONICET - Centro de Investigaciones Cuyo "Dr. Abelardo Pithod", Argentina \\ cdexposito@uncu.edu.ar
}

Recibido: 8/07/2020 - Aceptado: 5/11/2020

\section{Resumen}

El objetivo del presente estudio fue identificar las principales necesidades detectadas por docentes en su propio grupo de estudiantes durante el aislamiento social, preventivo y obligatorio dispuesto por el Gobierno Nacional en marzo de 2020. Participaron de esta investigación docentes y directivos de instituciones de distintos niveles educativos de Mendoza, Argentina ( $n=777)$. Los resultados cualitativos fueron trabajados y analizados a partir de las respuestas a preguntas abiertas y pequeñas narrativas que dan lugar a la libre expresión de los participantes. Mediante la Teoría Fundamentada se identificaron categorías explicativas que permitieron analizar necesidades que van más allá de las restricciones tecnológicas y de conectividad, entrando a la esfera económica, familiar, pedagógica y psicoafectiva de estudiantes de distintos contextos educativos. Dichos resultados constituyen una primera aproximación a los condicionantes que enfrenta el sistema educativo en tiempos de confinamiento social obligatorio. A su vez, constituyen un insumo relevante para la generación de conocimiento sobre la realidad educativa en esta época de pandemia y para la toma de decisiones a nivel de gestión institucional y provincial.

\footnotetext{
* Doctora en Educación, Profesora y Licenciada en Ciencias de la Educación por la Universidad Nacional de Cuyo. Especialista en Educación en Ambiente y Desarrollo Sustentable por la Universidad Nacional del Comahue. Investigadora Adjunta del Consejo Nacional de Investigaciones Científicas y Técnicas - CONICET. Docente de grado y posgrado en la Facultad de Ciencias Económicas de la Universidad Nacional de Cuyo en las áreas de Comportamiento Organizacional, Metodología de la Investigación y Taller de Tesis.

** Doctor en Educación y profesor de posgrado en la Facultad de Ciencias Económicas de la Universidad Nacional de Cuyo. Investigador del Centro de Investigaciones Cuyo "Dr. Abelardo Pithod" mediante una beca posdoctoral de CONICET en el área de Ciencias de la Educación y Psicología. Diplomado Universitario en Gestión Educativa y ha desempeñado tareas de asesoría para la Dirección General de Escuelas del Gobierno de Mendoza.
} 
ReVISTA DE LA ESCUELA DE CIENCIAS DE LA EduCACIÓN, AÑo 17, NRO. 16, VOL. 1, ENERO A JUNIO de 2021. PÁGINAS 1-10. ISSN 2362-3349 (EN LÍNEA). NUEVAS NECESIDADES EDUCATIVAS. UNA APROXIMACIÓN A LOS CONDICIONANTES DEL SISTEMA EDUCATIVO EN ÉPOCAS DE CONFINAMIENTO SOCIAL. ROXANA GRACIELA MARSOLLIER. CRISTIÁN DAVID EXPÓSITO.

Palabras clave: COVID-19 - Sistema Educativo - Docencia - Aislamiento Social - Necesidades Educativas - Gestión Educativa.

\title{
NEW EDUCATIONAL NEEDS AN APPROACH TO THE CONDITIONS OF THE EDUCATIONAL SYSTEM IN TIMES OF SOCIAL CONFINEMENT
}

\begin{abstract}
The objective of this study was to identify the main needs detected by teachers in their own group of students during the social, preventive and compulsory isolation ordered by the National Government in March 2020. Teachers and managers from different educational levels belonging to the province of Mendoza, Argentina $(n=777)$. The qualitative results were worked on and analyzed from the answers to open questions and small narratives that give rise to the free expression of the participants. By means of the Grounded Theory, explanatory categories were constructed that allowed identifying needs that go beyond technological and connectivity restrictions, entering the economic, family, pedagogical and psychoaffective sphere of students from different educational contexts. These results constitute a first approximation to the conditions that the educational system faces in times of compulsory social confinement. In turn, they constitute a relevant input for the generation of knowledge about the educational reality in this time of pandemic and for decision-making at the institutional and provincial management level.
\end{abstract}

Keywords: COVID-19 - Educational System - Teaching - Social Isolation - Educational Needs Educational Management.

\section{Introducción}

El reciente brote epidémico COVID-19 ha generado disrupciones educativas y colapsos en los sistemas de salud. Los países afectados se han visto en serias dificultades para poder enfrentar esta situación, ya que nadie es inmune y el mundo se siente abrumado por la velocidad de propagación y los efectos devastadores que deja a su paso. A pocos meses del primer caso, este virus ha cambiado profundamente el estilo de vida de toda la humanidad (Onyema, et al., 2020). El 3 de marzo se registra en Argentina el primer caso de COVID-19 y, 17 días más tarde, se decreta el aislamiento social preventivo y obligatorio para todo el país (Silva, 29 de abril de 2020), situación que obligó a toda la comunidad a tomar medidas para las cuales no estaba preparada. El sistema educativo en su totalidad se vio forzado a cerrar las puertas de todas las instituciones escolares a nivel presencial y reconvertirse en un modelo que trascienda el espacio físico. Ahora bien, esta trascendencia también debe darse desde lo simbólico en pos de brindar una educación que vaya más allá de lo establecido si no quiere perder su esencia, su sentido:

Debe ser un espacio de protección física, social y emocional para niños, niñas y jóvenes. De ahí viene su sentido. Un sentido que solo adquirirá su pleno valor si garantiza la adquisición de conocimientos profundos y relevantes para todos los estudiantes. Si actúa como espacio de inclusión -en sentido amplio- de todo el alumnado (Tarabini, 2020, s/p).

Por su parte, Kaplan y Szapu (2019) plantean que este 'lugar simbólico' que ocupan las escuelas tiene que estar en relación directa con su capacidad de inclusión democrática tratando de reducir las desigualdades socioculturales. La interrupción de clases fue abrupta y las políticas educativas que se aplicaron, muy variadas. "Buena parte de los países latinoamericanos suspendieron las clases e implementaron programas para mantener la escuela o la universidad 'a distancia'” (Dussel, 2020, p. 2). Junto al confinamiento de estudiantes y docentes, se construyó una red virtual que hace las veces de efector educativo entre los distintos actores del proceso. Por una parte, los docentes dejaron las aulas de lado para convertir sus espacios residenciales en aulas virtuales, teniendo que amigarse con una tecnología que les era totalmente prescindible antes de la 
ReVISTA DE LA ESCUELA DE CIENCIAS DE LA EduCACIÓN, AÑo 17, NRO. 16, VOL. 1, ENERO A JUNIO de 2021. PÁGINAS 1-10. ISSN 2362-3349 (EN LÍNEA). NUEVAS NECESIDADES EDUCATIVAS. UNA APROXIMACIÓN A LOS CONDICIONANTES DEL SISTEMA EDUCATIVO EN ÉPOCAS DE CONFINAMIENTO SOCIAL. ROXANA GRACIELA MARSOLLIER. CRISTIÁN DAVID EXPÓSITO.

cuarentena. Por otra parte, los estudiantes se encuentran confinados en sus hogares compartiendo dispositivos digitales con toda la familia (Sánchez, et al., 2020). Estas herramientas no siempre están disponibles y es en estas circunstancias donde se evidencian las desigualdades sociales, porque las familias con mayores recursos económicos son las que tienen más posibilidades de suplir el cierre de las instituciones educativas (Rodríguez, 2020). Sin embargo, los sectores más vulnerables encuentran en la escuela el apoyo pedagógico necesario junto a la posibilidad de satisfacer necesidades básicas tales como la alimentación mediante los comedores escolares (Álvarez et al., 2020). Esta situación no sólo es exclusiva de Argentina o de los países latinoamericanos, también Estados Unidos la padece en cierta medida (Holcombe, 2020).

El confinamiento obligatorio puso en evidencia las enormes desigualdades de conectividad en nuestro país (Dussel, 2020). Mendoza, puntualmente, consta de una cobertura del $72,2 \%$ para estudiantes de $7^{\circ}$ grado de nivel primario y del $79 \%$ para los de $5^{\circ}$ año de nivel secundario, según el reporte del Observatorio Argentinos por la Educación (Artopoulos, 2020). Sin embargo, los entornos de aprendizaje en línea generalmente requieren computadoras y una conexión a Internet confiable, porque de nada sirve la conectividad sin la tecnología adecuada para poder llegar a los hogares de los estudiantes; por lo tanto, se vuelve imperioso cubrir ambas demandas para acortar la brecha digital ${ }^{1}$ (Petretto, Masala, \& Masala, 2020; Espada, et al., 2020). Van Lancker y Parolin (2020) al igual que Dussel (2020) plantean que esta brecha repercute negativamente en el aprendizaje de estudiantes de bajos recursos ya que, en sus hogares particulares se pierden ciertas garantías y estándares educativos que brinda la escuela como institución. Puesto que, además de la conectividad y la tecnología, también existen algunos factores de confort que un colegio brinda a sus estudiantes para que puedan llevar a cabo su labor académica y que ciertos hogares por debajo de la línea de la pobreza carecen como calefacción, espacios y mobiliarios adecuados, inclusive aquellas comodidades hueras como un patio de recreación o, más imprescindibles, como un baño, electricidad o agua potable (Paz, 2020).

Otro factor que la educación a distancia no puede asumir totalmente es la contención emocional que cada docente brinda a sus estudiantes. Es muy diferente el tipo de relaciones sociales y el flujo de emociones que se producen en una instancia presencial física (Maldonado, 2020). Es necesario comprender que la enseñanza online no se refiere solo a la mera carga y descarga de documentos en formato pdf o docx, (Estrada, et al., 2015), implica también asumir el rol loable de agente motivador del aprendizaje incentivando aquellas habilidades de estudio, tales como:

...aprender a aprender, operar en el uso de estrategias de aprendizaje, manejar los organizadores de ideas para sintetizar y apropiarse de las competencias de lectura crítica para deducir lo realmente valioso de la información, mitigando de este modo los efectos del estrés y la frustración (Morales, 2020, p. 7).

Esta acción cotidiana, propia del docente que tiene una mirada integral de la educación, resulta muy difícil de practicar a la distancia. Sin embargo, es necesario el manejo de competencias basadas en estrategias de afrontamiento, reflexión, asertividad, empatía, escucha activa y contenedora para poder lograr el objetivo de cada actividad pedagógica (Villafuerte, et al., 2020).

Otra variable importante a considerar es el apoyo de la familia, si realmente se pretenden alcanzar los objetivos trazados en educación online. Las etapas inferiores de escolarización implican períodos sensibles respecto del proceso evolutivo-educativo del estudiante y demandan el acompañamiento de un familiar que haga las veces de tutor para el acceso y seguimiento de dicho proceso (Failache, Katzkowicz \& Machado, 2020). Tanto la escuela como el docente deben entablar un sólido vínculo con las familias de sus estudiantes para viabilizar el arte de enseñar, sin perder de vista que es algo positivo el desarrollo de tareas sincrónicas, motivantes y afectivas (Pérez, 2017). Sostienen Moreno y Molins (2020) que: "Las familias, en esta crisis sanitaria, pasan a ser un agente educativo de primer orden y fuente de aprendizaje. Constituyen un nivel imprescindible de colaboración con la educación y la consecución de las metas educativas" (p. 7). De esta manera llevan a cabo una actividad de apoyo y promueven un mejor desarrollo de los procesos de aprendizaje de sus hijos. Ahora bien, esta tarea no es fácil para todos los hogares, al margen de las falencias de conectividad o de equipamiento para la gestión de la educación en casa, la situación económica familiar es otra variable que influye negativamente (Bonavida \& Gasparini, 2020; Ríos Sierra, 2020).

Este estudio tiene por objeto aproximarnos a estas complejas situaciones a través de la voz de los docentes, quienes han podido identificar las principales necesidades que afrontan sus estudiantes en épocas de confinamiento social. La riqueza de sus respuestas radica en la mirada holística de una nueva realidad cotidiana, incipiente y desconocida, contextualizada en la relación con sus estudiantes de diferentes escuelas y regiones de la provincia de Mendoza.

${ }^{1}$ Nota del Autor: Cabe destacar que la Dirección General de Escuela (DGE), frente a esta realidad, desarrolló una campaña denominada "Tu ayuda nos conecta" (10 de abril de 2020). El objetivo de esta campaña es recaudar dinero para comprar teléfonos móviles y celulares usados en buen estado para distribuirlos a través de la Fundación Fonbec (http://www.fonbec.org.ar/) a los estudiantes, con el objeto que puedan finalizar satisfactoriamente sus estudios. 
REVISTA DE LA EsCuela de CIENCIAS DE LA EdUCACIÓN, AÑo 17, NRO. 16, VOL. 1, ENERO A JUNIO DE 2021. PÁGINAS 1-10. ISSN 2362-3349 (EN LÍNEA). NUEVAS NECESIDADES EDUCATIVAS. UNA APROXIMACIÓN A LOS CONDICIONANTES DEL SISTEMA EDUCATIVO EN ÉPOCAS DE CONFINAMIENTO SOCIAL. ROXANA GRACIELA MARSOLLIER. CRISTIÁN DAVID EXPÓSITO.

\section{Método}

\section{Diseño}

Se trabajó con un diseño cualitativo, parte de un estudio más amplio que también contempló una instancia cuantitativa. Los resultados que se abordan en esta comunicación surgen del análisis de las respuestas a preguntas abiertas y pequeños relatos, referidos a las necesidades detectadas por los docentes en su grupo particular de estudiantes. A partir de las argumentaciones vertidas, se trabajó en la construcción de categorías explicativas que permitieron reagrupar las principales falencias detectadas en cada uno de los niveles del sistema educativo.

\section{Participantes}

Colaboraron docentes y directivos de todos los niveles educativos de la Provincia de Mendoza, Argentina, en un total de 777 participantes. Según el nivel educativo, la mayor contribución está en educación de adultos $(36,16 \%)$, nivel secundario $(31,40 \%)$ y primario $(15,83 \%)$. En cuanto al tipo de gestión, el $87,91 \%$ de los participantes se desempeñan en instituciones de gestión pública. En cuanto a las variables sociodemográficas, hay un predominio de mujeres $(79,51 \%)$, de estado civil casado $(51,35 \%)$ y que tienen hijos (76,19\%). A su vez, la edad promedio de los encuestados es de 43 años.

\section{Instrumentos}

Los datos fueron recolectados mediante un cuestionario semiestructurado, que incluyó una instancia de abordaje cualitativo para recuperar la voz de los protagonistas; donde, mediante preguntas abiertas y pequeños relatos, los docentes se explayaron en las necesidades que detectaron durante el periodo de confinamiento en su grupo específico de estudiantes.

\section{Procedimiento y análisis de datos}

Para el relevamiento se utilizó Google Forms $\AA$, donde el acceso al cuestionario estaba precedido por un formulario de consentimiento informado, que exponía el objetivo de la investigación y declaraba el anonimato y el resguardo del secreto estadístico. Dicho formulario se difundió mediante correo electrónico y a través de WhatsApp $\AA$, invitando a los participantes a reenviarlo a otros colegas, a fin de generar un muestreo intencional por bola de nieve.

Por otra parte, los datos cualitativos fueron obtenidos mediante preguntas abiertas a los docentes, buscando conocer las principales necesidades educativas detectadas. Se identificaron 1305 respuestas válidas que representan necesidades concretas de los estudiantes de distintos niveles y tipos de gestión. Dichas expresiones, fueron analizadas sistemáticamente mediante el método de Teoría Fundamentada, identificando las categorías explicativas que permiten interpretar en qué aspectos se concentran las principales problemáticas (Strauss \& Corbin, 2016).

\section{Resultados}

En este apartado analizaremos las respuestas a las preguntas abiertas del cuestionario, donde los participantes comentan las principales necesidades que observan en su grupo de estudiantes, durante la etapa de cuarentena y ante la aplicación del sistema virtual de educación. Debido a la amplia cantidad de contribuciones, se optó por efectuar una cuantificación según la frecuencia con que aparecen reiteradamente las distintas categorías. Los resultados indican que las principales necesidades se encuentran vinculadas a la conexión de internet o problemas de conectividad (33.18\%), ausencia de recursos tecnológicos apropiados para insertarse en la modalidad virtual (ya sea por carencia de los mismos o por obsolescencia de los aparatos) $(24.53 \%)$ a lo que se suma una situación económica apremiante $(10.40 \%)$. Por otra parte, se identificaron necesidades pedagógicas (26.58\%) y de apoyo familiar (4.40\%) tal como muestra el siguiente gráfico: 


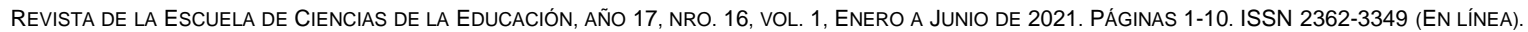
NUEVAS NECESIDADES EDUCATIVAS. UNA APROXIMACIÓN A LOS CONDICIONANTES DEL SISTEMA EDUCATIVO EN ÉPOCAS DE CONFINAMIENTO SOCIAL. ROXANA GRACIELA MARSOLLIER. CRISTIÁN DAVID EXPÓSITO.

Figura 1: Principales necesidades expresadas por los participantes

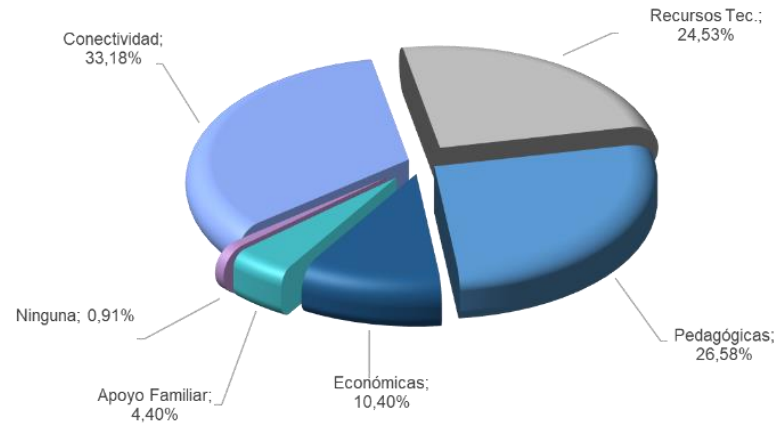

Fuente: Encuesta “Educación en tiempos de Pandemia" 2020

Como era de esperarse y de igual manera en todos los niveles educativos, los problemas de conectividad y de recursos tecnológicos han sido los más mencionados. Sin embargo, cada nivel educativo muestra el predominio de otro tipo de necesidades.

A continuación, desarrollamos las distintas categorías y ejemplificamos con las voces de los participantes.

\section{Categoría "Conectividad"}

Esta categoría toma su nombre como código in vivo, por la cantidad de veces que aparece el mismo término escrito en las respuestas y representa el $33.18 \%$ de los problemas detectados. A su vez, identificamos dos subcategorías: aquella que refiere exclusivamente a problemas de conectividad (mala señal, intermitencia, lejanía, irregularidad en la disponibilidad, etc.) y otra subcategoría que alude básicamente a la falta de acceso y/o conexión del servicio (ya sea por cuestiones económicas o por vivir el estudiante en zonas muy alejadas).

\section{Subcategoría "Dificultades de Conectividad"}

En cuanto a las necesidades de conectividad, la categoría satura rápidamente, es decir que las respuestas no varían demasiado, a lo que se suma la reiteración de dicho término. A modo de ejemplo, se pueden leer expresiones tales como:

"Dificultades con el funcionamiento de internet en las casas de los alumnos (y de profesores) que hacen que no funcione bien la continuidad de la enseñanza" (Universitario, Id.725)

"La falta de conectividad es grave" (Superior no universitario, Id.141)

"Escasa conectividad, es una zona aislada, no hay óptima señal" (Ed. Adultos, Id.266)

"Hay problemas de conectividad por razones monetarias o de cobertura (utilizan el crédito del teléfono para conectarse, investigar y descargar material)" (Ed. Adultos, Id.322)

"Conectividad necesaria para recibir/enviar tareas o acceder a alguna plataforma o videollamada" (Secundario, Id.111)

"No tienen acceso a buena conectividad de internet, para muchos depende del crédito en su teléfono" (Primario, Id.023)

"Falta de conectividad en contextos rurales" (Primario, Id.144)

\section{Subcategoría "Falta de acceso a Internet"}

En menor medida, también se identifica la falta de conexión a internet, lo que observamos en respuestas tales como:

"No todos cuentan con acceso a internet por lo cual se complica el traspaso de información" (Superior no universitario, Id. 011)

"La mayoría no tiene conexión ni acceso posible a ella" (Ed. Adultos, Id.512)

"La principal necesidad es de acceso a conexiones estables de internet" (Secundario, Id.168)

"Algunos no poseen computadoras ni celulares y mucho menos internet" (Secundario, Id.643)

"No todos los estudiantes tienen acceso a la tecnología y sobre todo a internet" (Primario, Id.769) 
ReVISTA DE LA ESCUELA DE CIENCIAS DE LA EduCACIÓN, AÑo 17, NRO. 16, VOL. 1, ENERO A JUNIO de 2021. PÁGINAS 1-10. ISSN 2362-3349 (EN LÍNEA). NUEVAS NECESIDADES EDUCATIVAS. UNA APROXIMACIÓN A LOS CONDICIONANTES DEL SISTEMA EDUCATIVO EN ÉPOCAS DE CONFINAMIENTO SOCIAL. ROXANA GRACIELA MARSOLLIER. CRISTIÁN DAVID EXPÓSITO.

\section{Categoría "Recursos Tecnológicos"}

En esta categoría se agruparon aquellas respuestas que dan cuenta de los insumos técnicos e informáticos que necesita el grupo de estudiantes para poder acceder a las clases virtuales. Esta categoría representa un $24.53 \%$ del total de respuestas ofrecidas en relación con las necesidades de los estudiantes. La categoría satura rápidamente y se observan expresiones como las siguientes:

"No todos los alumnos cuentan con recursos tecnológicos, como teléfonos, computadoras, impresoras" (Ed. Adultos, Id.458)

"Recursos tecnológicos escasos o poco actualizados (computadora, celular)" (Ed. Adultos, Id.698)

"No tienen los medios tecnológicos necesarios para realizar los trabajos de forma virtual" (Ed. Adultos, Id.267)

"La escasez de recursos tecnológicos que permitan mayor y mejor vinculación durante las clases" (Ed.

Adultos, Id.640)

"Celulares sin memoria...falta de definición en imágenes" (Secundario, Id.635)

"No tienen los recursos tecnológicos para poder trabajar de forma online" (Secundario, Id.735)

"Muchos alumnos tienen problemas de comunicación por no contar con computadora, celular y acceso a internet" (Secundario, Id.101)

"La falta de celular, ya que, en muchos casos, poseen un celular por familia y tienen varios hijos en edad escolar" (Secundario, Id. 528)

\section{Categoría "Necesidades Económicas"}

Al reclamo por la falta de internet o conectividad o la escasez de recursos tecnológicos adecuados para la educación virtual, se suman las "Necesidades Económicas". Esta categoría representa un 10.40\% del total de respuestas ofrecidas. Se observó particularmente en Nivel Secundario y Educación de Adultos. Dentro de la categoría se han identificado en su mayoría problemas referidos a la situación económica familiar que se manifiestan en frases tales como:

"La mayoría son de familias de recursos económicos y sociales muy humildes" (Ed. Adultos, Id.706)

"Van a poder comunicarse hasta cuando se puedan pagar el crédito para el teléfono, que por ahora tienen de lo que ganaron en la época de cosecha, ya que en la zona en la que viven es muy alejada y no tienen conexión por antena sólo por crédito" (Ed. Adultos, Id.434)

"Necesidades económicas que imposibilita el acceso a la tecnología" (Ed. Adultos, Id.270)

"Al trabajar con grupos socioeconómicos bajos el principal método de trabajo es WhatsApp y el mismo no permite un desarrollo muy amplio de las consignas" (Ed. Adultos, Id.240)

"Falta de dinero para adquirir paquete de datos" (Secundario, Id.711)

"Falta de recursos digitales y económicos" (Secundario, Id.690)

"Enfrentan situaciones económicas difíciles" (Secundario, Id.357)

"Desde lo económico no se puede acompañar a los chicos con el material didáctico" (Primario, Id.117)

“...son niños de zonas muy vulnerables y no disponen de recursos económicos. Se trató de explicar a las familias que trabajaran con material que tuvieran en casa y reciclaran y utilizaran otros elementos para realizar las actividades" (Inicial, Id.138)

Sin embargo, un margen aproximado del $20 \%$ del total de respuestas en la categoría de "Necesidades Económicas", refiere a necesidades básicas insatisfechas identificadas sobre la situación laboral de la familia, condiciones de vivienda y alimentación. Así lo expresan las siguientes frases:

"Necesidades de alimentación, casa digna, trabajo" (Ed. Adultos, Id.067)

"Búsqueda de ingresos, trabajo" (Ed. Adultos, Id.518)

"También tienen necesidades básicas insatisfechas. Hacinamiento en algunos casos" (Secundario, Id.340)

"Necesitan comida porque muchos padres no pueden trabajar y no tienen salario" (Secundario, Id.566)

"Algunos sufrieron cambios económicos (desempleo, falta de ingresos)" (Secundario, Id.643)

"Pobreza estructural" (Secundario, Id.179)

"La situación laboral de los padres" (Primario, Id.651)

"La falta de condiciones sociales y económicas. Muchas personas conviviendo en lugares muy pequeños y con falta de comodidades para realizar las actividades" (Primario, Id.189)

\section{Categoría "Necesidades Pedagógicas"}

Analizamos a continuación las "Necesidades pedagógicas" que representan el $26.58 \%$ de las respuestas. Esta categoría pone en evidencia que la docencia y el acto educativo va mucho más allá de la enseñanza y aprendizaje de contenidos. Así, esta categoría ha sido dividida en tres subcategorías: necesidades 
REVISTA DE LA EsCuela de CIENCIAS DE LA EdUCACIÓN, AÑo 17, NRO. 16, VOL. 1, ENERO A JUNIO DE 2021. PÁGINAS 1-10. ISSN 2362-3349 (EN LÍNEA). NUEVAS NECESIDADES EDUCATIVAS. UNA APROXIMACIÓN A LOS CONDICIONANTES DEL SISTEMA EDUCATIVO EN ÉPOCAS DE CONFINAMIENTO SOCIAL. ROXANA GRACIELA MARSOLLIER. CRISTIÁN DAVID EXPÓSITO.

socioafectivas, dificultades propias de los procesos de enseñanza-aprendizaje y dificultades referidas a la implementación de la modalidad virtual.

\section{Subcategoría "Necesidades Socio-afectivas"}

La subcategoría de necesidades socio-afectivas aparece en todos los niveles, con mayor frecuencia en educación de adultos, nivel inicial y primaria. Así, los docentes señalan:

"La necesidad del contacto social" (Universitario, Id.196)

"Más feedback entre docentes y alumnos" (Superior no universitario, Id.732)

"El contacto cara a cara por un sistema de vídeo llamada" (Superior no universitario, Id.068)

"Contacto. Hoy me dijeron que extrañan charlar en el recreo" (Ed. Adultos, Id.048)

"Es trascendental la interacción entre personas" (Ed. Adultos, Id.523)

"La necesidad de la contención que se da en el aula" (Ed. Adultos, Id.176)

"La necesidad del vínculo, mis alumnos son adultos mayores" (Ed. Adultos, Id.273)

"Acompañamiento constante, docente guía y mediadora del acto educativo" (Ed. Adultos, Id.232)

"Necesitan el contacto con el docente para poder construir un aprendizaje realmente significativo" (Secundario, Id.167)

"La necesidad de las clases presenciales ninguna tecnología las suple" (Secundario, Id.468)

(necesidad de) "Ser escuchados y tener al docente a su lado" (Secundario, Id.599)

"Poco tiempo para establecer el vínculo fundamental para avanzar en el proceso de enseñanza/aprendizaje" (Primario, Id.594)

"Necesidad de compartir sus emociones" (Primario, ld.571)

(Necesidades) "Ligadas a lo emocional y a la necesidad de vincularse y ser visualizado específicamente por sus docentes" (Primario, Id.663)

"La necesidad de relacionarse con sus pares, sus maestros" (Primario, Id.677)

"El aprendizaje que se logra con el grupo de pares" (Inicial, Id.031)

"El contacto y vínculo con la seño" (Inicial, Id.084)

\section{Subcategoría "Necesidades de enseñanza y aprendizaje”}

En relación con la subcategoría que identifica las necesidades asociadas con el proceso de enseñanza y aprendizaje, aparece especialmente en el nivel medio, y se pone de manifiesto en expresiones tales como:

"No saben estudiar solos" (Universitario, Id.001)

"Falta de organización del tiempo de estudio" (Superior no universitario, Id.185)

"Las consignas de trabajo deben ser mucho más claras y definidas que en las clases presenciales. Se producen fácilmente malos entendidos" (Superior no universitario, Id.399)

"Reciben el material, envían las tareas por imágenes, pero no hay intercambio en WhatsApp de dudas, consultas, etc." (Secundario, Id.096)

(necesitan) "Interpretación de consignas y comprensión de texto" (Secundario, Id.076)

(es necesario) "Explicar una y otra vez los temas" (Secundario, Id.286)

(necesitan) "Mayor comprensión lectora. Prefieren audios o videos" (Secundario, Id.308)

"Académicamente necesitan mayor autonomía y comprensión de texto" (Secundario, Id.259)

(necesitan) "Práctica de la habilidad para comunicarse oralmente" (Secundario, Id.025)

"Las dificultades de establecer rutinas de trabajo, la autonomía, hábitos de estudio" (Primario, Id.075)

"Apoyo pedagógico para la realización de las tareas" (Primario, Id.208)

"Comprensión lectora" (Primario, Id.053)

"Falta interpretación de consignas" (Inicial, Id.046)

\section{Subcategoría "Modalidad virtual"}

Finalmente, en relación con la subcategoría de necesidades detectadas en el manejo de la modalidad virtual, las respuestas ponen en evidencia una falta de adecuación desde todos sus actores. Comenzando por las instituciones, la escasa capacitación docente y las dificultades en el manejo de recursos virtuales por parte de los estudiantes, ya que los participantes detectan:

"Dificultades con funcionamiento de plataformas institucionales" (Universitario, Id.725)

"Principalmente la dificultad vino de la institución educativa, no estaba preparada y demoró bastante en incluir a los alumnos a la plataforma virtual" (Superior no universitario, Id.158)

"La falta de capacitación docente para mediar clases a distancia" (Secundario, Id.207) 
ReVISTA DE LA ESCUELA DE CIENCIAS DE LA EduCACIÓN, AÑo 17, NRO. 16, VOL. 1, ENERO A JUNIO de 2021. PÁGINAS 1-10. ISSN 2362-3349 (EN LÍNEA). NUEVAS NECESIDADES EDUCATIVAS. UNA APROXIMACIÓN A LOS CONDICIONANTES DEL SISTEMA EDUCATIVO EN ÉPOCAS DE CONFINAMIENTO SOCIAL. ROXANA GRACIELA MARSOLLIER. CRISTIÁN DAVID EXPÓSITO.

Especialmente, en educación secundaria y en el nivel superior también, aunque en menor medida, se evidencia un reclamo de los docentes por la falta de manejo de recursos tecnológicos por parte de sus estudiantes, quienes no se adaptaron tan fácilmente al sistema educativo virtual. Algunas razones:

"No están familiarizados con la modalidad online" (Universitario, Id.318)

"Falta de capacitación en el uso de los recursos, asumimos que nuestros estudiantes son nativos informáticos, pero solo saben de usar Instagram y sacarse fotos" (Superior no universitario, Id.670) (necesidad de) "Capacitación en manejo de plataformas y contenidos digitales" (Superior no universitario, Id.078)

"Carencia de...manejo de recursos digitales en educación" (Secundario, Id.156)

"Nulo manejo de correo electrónico y app de edición de documentos digitales. Poca predisposición ante nuevos desafíos tecnológicos" (Secundario, Id.509)

"También el nulo manejo de mail o plataformas como GoSchool" (Secundario, Id.255)

"No manejan herramientas básicas como Word, envío de mail, interpretación de un tutorial" (Secundario, Id.767)

"No saben utilizar herramientas tecnológicas, no pueden ingresar a la plataforma o no saben utilizar un PowerPoint" (Secundario, Id.251)

"Conocimientos básicos de correo y de Word. Los mal llamados "nativos digitales" no saben cómo enviar un e-mail o cómo configurar márgenes de una hoja" (Secundario, Id.062)

"Los adolescentes no manejan las TIC's. Sólo usan redes sociales lo que hace que se desanimen al presentar los trabajos. Los consideran imposibles de hacer, pero es que no saben manejar un pdf, por ejemplo" (Secundario, Id.776)

\section{Categoría "Apoyo familiar"}

Por último, destacamos la categoría que agrupa aquellas necesidades de apoyo familiar en este periodo de incertidumbre. El acompañamiento de la familia ha sido especialmente reseñado en educación primaria y secundaria, tal como lo ilustran los siguientes comentarios:

"En el secundario veo la necesidad de que los padres tengan un mejor seguimiento de las tareas de sus hijos" (Secundario, Id.583)

"Falta de acompañamiento por parte de la familia" (Secundario, Id.701)

"Falta de capital cultural familiar para ayudar" (Secundario, Id.002)

"La falta de conocimiento de herramientas tecnológicas por parte de los padres para poder ayudarlos en las tareas" (Primario, Id.189)

"Empezaron con entusiasmo y con el tiempo se hace más difícil que los padres sean comprometidos con la enseñanza de los chicos" (Primario, Id. 006)

(Necesidad de) "Compromiso de la familia para juntos poder llevar adelante la enseñanza a distancia" (Primario, ld. 007)

"Al no estar asistiendo al edificio escolar disminuye la responsabilidad familiar" (Primario, Id. 052)

"Falta de educación de los padres que les impide ayudar a sus hijos" (Primario, Id.592)

En síntesis, teniendo en cuenta estos pequeños relatos de los participantes organizados en diferentes categorías podemos apreciar mejor una lectura de la nueva normalidad, ya que son los docentes quienes están en contacto directo y cotidiano con los estudiantes. Este estudio tiene una visión integral de los distintos condicionantes del sistema educativo regional en tiempos de pandemia.

\section{Aproximaciones finales}

Nuestro objetivo fue aproximarnos a la nueva realidad educativa en tiempos de confinamiento social a fin de identificar las principales necesidades detectadas por docentes y directivos de distintos niveles educativos en su propio grupo de estudiantes.

Teniendo en cuenta el estudio cualitativo llevado adelante, los participantes plantearon una serie de necesidades donde la conectividad fue la más reclamada, seguido de la escasez de recursos tecnológicos por parte de los educandos. Una de las categorías que queremos resaltar en este trabajo es el aspecto pedagógico por la riqueza y variedad de matices en las respuestas; tal es así que su abordaje nos instó a realizar subcategorizaciones de la misma. Nos llamó poderosamente la atención la demanda afectiva por parte de la educación en adultos, si bien era un aspecto que esperábamos se evidenciara en niveles de educación inicial y primario, los docentes de adultos manifestaron la importancia que tiene el aspecto socio-afectivo para el ejercicio de la profesión en este nivel. También pudimos adentrarnos en aquellos problemas propios del proceso de enseñanza y aprendizaje, lo que puso en evidencia, principalmente en los docentes del nivel secundario, una serie de reclamos por dificultades en la comprensión lectora por parte de sus estudiantes. La última de las 
ReVISTA DE LA ESCUELA DE CIENCIAS DE LA EduCACIÓN, AÑo 17, NRO. 16, VOL. 1, ENERO A JUNIO de 2021. PÁGINAS 1-10. ISSN 2362-3349 (EN LÍNEA). NUEVAS NECESIDADES EDUCATIVAS. UNA APROXIMACIÓN A LOS CONDICIONANTES DEL SISTEMA EDUCATIVO EN ÉPOCAS DE CONFINAMIENTO SOCIAL. ROXANA GRACIELA MARSOLLIER. CRISTIÁN DAVID EXPÓSITO.

subcategorías que se desprendió de esta dimensión refiere a limitaciones en la implementación de la modalidad virtual; tanto a nivel de instituciones que no contaban con la infraestructura tecnológica necesaria, como de estudiantes que presentaron dificultades en el uso de esta modalidad, especialmente en el nivel secundario.

Respecto de la categoría que unificó las demandas económicas pudimos observar que se acentuaba especialmente en los testimonios de los docentes que se desempeñan en el nivel secundario y de adultos. Esta dimensión aportó dos subcategorías, la más relevante fue la referida al acceso de la tecnología ya que muchos de ellos relataron que la educación de sus estudiantes, no solo dependía de la posibilidad de acceso a internet o de comprar a un paquete de datos, sino que también estaba condicionada por el acceso al teléfono celular de un padre o hermano. En menor medida, la otra subcategoría que incluye esta dimensión económica, se relaciona con las Necesidades Básicas Insatisfechas y todo lo que ellas implican desde el hacinamiento, falta de trabajo, vivienda, alimento, etc., aspectos que ponen en evidencia las desigualdades sociales.

En síntesis, los resultados obtenidos confirman las primeras aproximaciones efectuadas en otros contextos, que dan cuenta de que la situación de pandemia puso en evidencia la desigualdad de oportunidades educativas, las marcadas disparidades entre quienes tuvieron mejor acceso a los recursos tecnológicos y a internet; las diferencias en el capital cultural de las familias, entre otros aspectos. En un país democrático, la inequidad en la educación solo denuncia injusticia social. Sin embargo, hay algo positivo que podemos extraer de una situación de catástrofe, como se nos está presentando esta pandemia mundial, y es que nadie puede sobrevivir de manera individual. Tenemos que trabajar mancomunadamente o la batalla ya estará perdida antes de darla. La clave está en los valores humanos como la solidaridad, la fraternidad y el compromiso. Cabe señalar que de ninguna manera este llamado concierne exclusivamente a los docentes, todas las profesiones y personas de nuestro país y el mundo tienen una obligación con la misma Humanidad (Centeno Hurtado, 2020)

\section{Referencias bibliográficas}

Álvarez, M.; Gardyn, N.; lardelevsky, A. \& Rebello, G. (2020). Segregación Educativa en Tiempos de Pandemia: Balance de las Acciones Iniciales durante el Aislamiento Social por el COVID-19 en Argentina. En: Revista Internacional de Educación para la Justicia Social, 9(3). DOI: https://doi.org/10.15366/riejs2020.9.3.002

Artopoulos, A. (2020). ¿Cuántos estudiantes tienen acceso a Internet en su hogar en Argentina? Observatorio Argentinos por la Educación. Disponible en: https://argentinosporlaeducacion.org/informes

Bonavida, C. \& Gasparini, L. (2020). El impacto asimétrico de la cuarentena. Documentos de Trabajo del CEDLAS. Disponible en: http://www.cedlas.econo.unlp.edu.ar/wp/

Centeno Hurtado, K. (2020). De La Peste al Coronavirus: sobre la virtud de lo heroico en tiempos de pandemia. En: Revista Salud UIS, 52(2), pp.178-179. Disponible en: https://revistas.uis.edu.co/index.php/revistasaluduis/article/view/10931

Dussel, I. (2020). La escuela en la pandemia. Reflexiones sobre lo escolar en tiempos dislocados. En: Práxis Educativa, 15, 1-16. Disponible en: https://doi.org/10.5212/PraxEduc.v.15.16482.090

Espada, J. P.; et al. (2020). Las buenas prácticas en la atención psicológica infanto-juvenil ante el COVID-19. En: Clínica y Salud. Avance online. https://doi. org/10.5093/clysa2020a14.

Estrada, V.; et al. (2015). La educación virtual. Diseño de cursos virtuales. Editorial Universidad ECOTEC

Failache, E.; Katzkowicz, N; \& Machado, A. (2020). La educación en tiempos de pandemia. Y el día después. Aportes y análisis en tiempos de coronavirus. En: Departamento de Economía, URU. https://www.colibri.udelar.edu.uy/jspui/bitstream/20.500.12008/24008/1/La_educaci\%C3\%B3n_en_tiempos _de_pandemia._Y_el_d\%C3\%ADa_despu\%C3\%A9s.pdf

Holcombe, M. (18 de marzo de 2020). Some schools closed for coronavirus in US are not going back for the rest of the academic year - CNN. CNN.Com. https:/edition.cnn.com/2020/03/18/us/coronavirus-schools-not-goingback-year/index.html

Kaplan, C. V., \& Szapu, E. (2019). Jóvenes y subjetividad negada: Apuntes para pensar la intervención socioeducativa sobre prácticas autolesivas y suicidio. En: Psicoperspectivas, 18(1), 42-52. https://scielo.conicyt.cl/scielo.php?pid=S0718-69242019000100042\&script=sci_arttext

Maldonado, R. (2020). La Intervención Social en tiempos de Pandemia. Reflexiones desde el Trabajo Social. Dosier de Actualidad. En: Revista Margen. https://www.margen.org/pandemia/textos/maldonado.pdf

Morales, J. (2020). Oportunidad o Crisis Educativa: Reflexiones desde la Psicología para Enfrentar los Procesos de Enseñanza-Aprendizaje en Tiempos de COVID-19. En: Revista Internacional de Educación para la Justicia Social, 9(3). https://revistas.uam.es/riejs/article/view/12228

Moreno, J. L. M., \& Molins, L. L. (2020). Educación y Covid-19: Colaboración de las Familias y Tareas Escolares. En: Revista Internacional de Educación para la Justicia Social, 9(3). https://dialnet.unirioja.es/servlet/articulo?codigo=7423226

Onyema, E. M.; et al. (2020). Impact of Coronavirus Pandemic on Education. En: Journal of Education and Practice. 11(13) 108-121. DOI: 10.7176/JEP/11-13-12.

Paz, J. (2020). La pobreza y la desigualdad de niñas, niños y adolescentes en la Argentina. Efectos del COVID-19. Disponible en: UNICEF Argentina. http://www.unicef.org/argentina/informes/pobreza-desigualdad-infantilCOVID19. 
ReVISTA DE LA ESCUELA DE CIENCIAS DE LA EduCACIÓN, AÑo 17, NRO. 16, VOL. 1, ENERO A JUNIO de 2021. PÁGINAS 1-10. ISSN 2362-3349 (EN LÍNEA). NUEVAS NECESIDADES EDUCATIVAS. UNA APROXIMACIÓN A LOS CONDICIONANTES DEL SISTEMA EDUCATIVO EN ÉPOCAS DE CONFINAMIENTO SOCIAL. ROXANA GRACIELA MARSOLLIER. CRISTIÁN DAVID EXPÓSITO.

Pérez, J. (2017). El desarrollo afectivo según Jean Piaget [en línea]. En: Revista vinculando. http://vinculando.org/psicologia_psicoterapia/desarrollo-afectivo-jean-piaget.html

Petretto, D. R.; Masala, I. \& Masala, C. (2020). Special Educational Needs, Distance Learning, Inclusion and COVID19. En: Education Sciences, 10(154). doi:10.3390/educsci10060154

Ríos Sierra, J. (2020). La inexistente respuesta regional a la COVID-19 en América Latina. En: Geopolítica(s). Revista De Estudios Sobre Espacio Y Poder, 11 (Especial), 209-222. DOI: https://doi.org/10.5209/geop.69324

Rodríguez, R. (2020). La desigualdad ante el espejo del COVID-19. En: Moya et al. (2020) La Psicología Social ante el COVID19: Monográfico del International Journal of Social Psychology. Revista de Psicología Social. DOI: https://doi.org/10.31234/osf.io/fdn32

Sánchez, M. et al. (2020). Retos educativos durante la pandemia de COVID-19: una encuesta a profesores de la UNAM. En: Revista Digital Universitaria, 21(3). http://doi.org/10.22201/codeic.16076079e.2020.v21n3.a12

Silva, D. (29 de abril de 2020). Cronología del coronavirus: más de 4.100 contagios tras 40 días de cuarentena. En: Perfil. https://www.perfil.com/noticias/coronavirus/pandemia-COVID19-cronologia-del-coronavirus-enargentina-tiempo-real.phtml

Strauss, A. \& Corbin, J. (2016). Bases de la investigación cualitativa: técnicas y procedimientos para desarrollar la teoría fundamentada. Universidad de Antioquia.

Tarabini, A. (2020). ¿Para qué sirve la escuela? Reflexiones sociológicas en tiempos de pandemia global. Revista de Sociología de la Educación-RASE, 13(2), 145-155. DOI: http://dx.doi.org/10.7203/RASE.13.2.17135

Tu Ayuda Nos Conecta (10 de abril de 2020). En: Prensa DGE. http://www.mendoza.edu.ar/tuayudanosconecta/

Van Lancker, W., \& Parolin, Z. (2020). COVID-19, school closures, and child poverty: a social crisis in the making. En: The Lancet Public Health, 5(5), e243-e244. DOI: https://doi.org/10.1016/S2468-2667(20)30084-0

Villafuerte, J. et al. (2020). Rol de los docentes ante la crisis del COVID-19, una mirada desde el enfoque humano. En: REFCalE: Revista Electrónica Formación y Calidad Educativa. 8(1), 134-150. http://www.refcale.uleam.edu.ec/index.php/refcale/article/view/3214 